\title{
DIANA AUGUSTA Y EL COLLEGIUM LOTORUM (CIL XIV 2156) LOS PIACULA DEL EMPERADOR CLAUDIO*
}

\author{
María José Pena \\ Universitat Autònoma de Barcelona \\ sideramundivaga@telefonica.net
}

\section{RESUMEN}

Estudio de una inscripción del s. I hallada en Ariccia (Lacio) y dedicada a Diana por dos curatores de un collegium lotorum, cuyas funciones se desconocen. Análisis del término lotores. Posible relación con otra inscripción del santuario de Diana en Nemi (en el término de Ariccia) y con los piacula celebrados en el año 50 por orden del emperador Claudio. Consideraciones sobre la purificación por el agua.

Palabras Clave: lotores, Diana, Nemi, piacula.

\author{
DIANA AUGUSTA AND THE COLLEGIUM LOTORUM (CIL XIV 2156) \\ THE PIACULA OF EMPEROR CLAUDIUS
}

\section{ABSTRACT}

Study of an inscription from the $1^{\text {st }}$ century found in Ariccia (Latium) and dedicated to Diana by two curatores of a collegium lotorum, whose functions are unknown. Analysis of the term lotores. Possible relation to another inscription from the sanctuary of Diana at Nemi (within the Ariccia municipal area) and to the piacula held in the year 50 by order of Emperor Claudius. Observations on the purification by water.

KeYwords: lotores, Diana, Nemi, piacula.

El trabajo que aquí presento pretende rendir merecido homenaje al amigo Ángel, que tan cordial y afectuoso ha sido siempre conmigo; lamento no poder ofrecer algún hermoso epigrama griego a quien ha dedicado buena parte de su trabajo y de su vida a la epigrafía cretense; no obstante, aunque esté escrito en latín y sea poco poético, no saldremos del mundo epigráfico y analizaremos un texto hallado en un santuario del Lacio, un lugar tan sugerente que inspiró a Sir J. G. Frazer el inicio de su famosa Rama dorada. El escenario natural del episodio que vamos a comentar es, entonces como ahora, uno de los parajes más bellos y atractivos de los Colli Albani; el lago de Nemi, situado al sureste de Roma, está rodeado por un bosque de encinas, castaños y otras especies; allí, ubicado en su ribera norte, el muy antiguo santua- 
rio de Diana atraía a devotos de toda índole, incluidas mujeres encintas, políticos, poetas..., y también emperadores. El santuario estaba en territorio del municipium aricinum; Aricia, la primera statio de la vía Apia (Hor. Sat. 1.5.1-2. Egressum magna me accepit Aricia Roma / hospitio modico), a XVI millas de Roma, era una de las antiguas e ilustres ciudades de la Liga Latina. Para quienes no conozcan el lugar, conviene aclarar que Ariccia no está situada a orillas del lago (que ni siquiera se ve desde la población) y que dista unos $4 \mathrm{~km}$. del santuario. Sobre él sabemos bastante, tanto a nivel literario como arqueológico, aunque en realidad sabemos muy poco; a excepción del ritual del rex nemorensis, apenas conocemos su funcionamiento, sus ceremonias, su personal o su administración; por los versos de algunos poetas sabemos que en los idus de agosto se celebraba una procesión de antorchas: Prop. 2.32.9-10, cum videt accensis deuotam currere taedis / in nemus et Triviae lumina ferre deae, Ov. Fast. 3. 270, Femina lucentes portat ab Urbe faces, Stat. Sil. 3.1.56-60, Fumat Aricinum Triviae nemus et face multa / conscius Hippolyti splendet lacus; ... Itala terra focis Hecateidas excolit idus. Estas referencias encajan sin problema con las numerosas imágines de Diana Lucifera, portadora de una (o dos) antorcha, que encontramos en la estatuaria o en la numismática (por ejemplo RRC 494/22 y 23; año 42 a.C.). El día de la fiesta de Diana en Nemi era el mismo que el de la fiesta de Diana en el Aventino, santuario que según la tradición literaria (Liv. 1.46; D.H. 4.26.5) había sido fundado por el rey Servio Tulio a mediados del s. VI a.C. (Pena, 1973; Malaspina, 1996; Scodellari, 2003); el día de esta festividad lo tenemos testimoniado por uno de los fragmentos de los Fasti Antiates, datados en el segundo tercio del s. I a.C. y también por Festo 467 L, Servorum dies festus erat Idibus Augusti, quia eo die rex Tullius, filius ancillae, aedem Dianae dedicavit. Aunque no lo parezca, esta circunstancia tiene su importancia ya que confirma que, en época imperial, ambos cultos se habían en cierto modo "unificado" y la festividad de Diana era sólo una, como muestra la lex del collegium Dianae et Antinoo (CIL XIV 2112, columna I, línea 5, [natali]s Dianae Idib(us) Aug(ustis), de Lanuvio datada en el año 133 (Garofalo, 2014).

En un trabajo anterior (Pena, 2018) dediqué mi atención al que es considerado como el primer hallazgo epigráfico realizado, a mediados del s. XVI, en el área del santuario de Diana y que ha resultado ser de dudosa autenticidad. Aquí y ahora presento un estudio y la consecuente reflexión sobre el que sería el primer hallazgo epigráfico, también en el s. XVI, realizado en Ariccia. Hay una distinción entre santuario y municipio.

* Este trabajo ha sido realizado en el marco del Proyecto de Investigación del Ministerio de Economía y Competitividad FFI2016-79906-P, titulado "Estudio diacrónico de las instituciones sociopolíticas de la Grecia antigua y de sus manifestaciones míticas", dirigido por el prof. Carlos Varias (UAB), a quien agradezco, así como también al prof. Xavier Espluga (UB), sus ayudas bibliográficas en tiempos complicados para todos y para todo. 


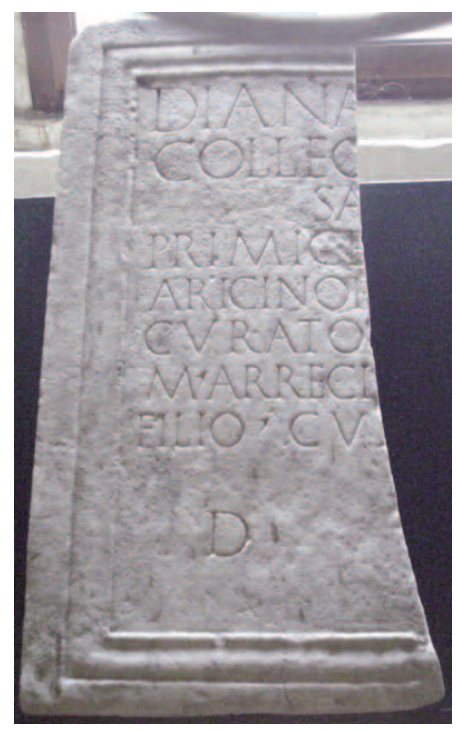

Fig. 1: CIL XIV 2156 (palacio Chigi, Ariccia).

Foto: M. J. Pena.

\section{LA INSCRIPCIÓN DE ARICIA CIL XIV 2156 = EDR143464 (FIG. 1)}

Se trata de una tábula de mármol blanco con moldura, de buena factura, conservada en una sala del palacio Chigi ${ }^{1}$, de Ariccia, donde pude estudiarla personalmente el 22 de febrero de 2017; está cortada en sentido vertical con una ligera curvatura. A finales del s. XVIII, Lucidi (1796: 84) todavía debió verla entera.

Medidas: grosor: $6 \mathrm{~cm}$., altura: $65 \mathrm{cms}$., anchura de lo conservado: $27 \mathrm{~cm}$. en la parte superior, $33 \mathrm{~cm}$. en la inferior. Letras de tamaño decreciente: $4^{\prime} 5 \mathrm{~cm} .1^{\mathrm{a}} \mathrm{l}$., 4 cm. 2al., 3'5 cm. Datada por Granino Cecere (2000: 38) en torno a la segunda mitad del s. I d.C.

\section{FECHA Y LUGAR DEL HALLAZGO}

Se desconocen la fecha y circunstancias precisas del hallazgo, pero poseemos un dato que permite una aproximación: en el manuscrito de su obra Libri delle iscrizioni latine e greche, conservado en Nápoles, Pirro Ligorio nos proporciona un dibujo, en el que aparece en forma de $\mathrm{ara}^{2}$; fue hallada por tanto antes de la composición

${ }^{1}$ Agradezco la colaboración, el interés y la amable acogida del Arch. Francesco Petrucci, Conservatore del palazzo Chigi in Ariccia.

${ }^{2}$ Ligorio (2008: 194), dice que esta dedicatoria está escrita dos veces, una en una base, como él dibuja, y otra en una tabula, pero esto lo hace en otras ocasiones. 
de su tratado Delle Antichità, vendido a Alejandro Farnese en 1567. No deja de ser interesante, y comprensible que algunos autores (p. ej. Guasco, 1775) la consideren un hallazgo posterior, pues el epígrafe debió estar empotrado en un muro dentro de la fortaleza de los Savelli (Petrucci, 2001) (propietarios del feudo de Ariccia desde 1423 hasta 1661, cuando lo compraron los Chigi) que fue parcialmente demolida para dejar paso a la construcción del actual palacio Chigi entre 1666 y 1672 bajo la dirección del arquitecto Carlo Fontana. Por tanto, transcurrió al menos un siglo entre el hallazgo y su "divulgación"; durante este tiempo, el texto, entonces completo, debió estar a la vista pero dentro de la propiedad Savelli y su buen estado de conservación hace pensar que no fue reutilizado como simple sillar pues la superficie estaría más estropeada. Pudiera ser que los materiales antiguos reutilizados en la fortaleza Savelli procedieran de algún monumento romano próximo al lugar, ubicado en la acrópolis aricina. Estas circunstancias son interesantes porque los hechos se ubican en Ariccia, no en Nemi, dos feudos distintos tanto en el s. XVI como durante los siglos posteriores. En la época que nos ocupa, Nemi, en la orilla opuesta del lago, pertenecía a los Colonna, que lo vendieron a los Frangipane en 1572. Así que no se puede afirmar que el epígrafe procede del santuario de Nemi, como hacen algunos autores (Diosono, 2011: 116). Siempre, durante siglos, ha estado (y sigue estando) en el feudo de Ariccia y este hecho proporciona otra perspectiva para su estudio y plantea la cuestión de las relaciones "funcionales" y quizás también económicas entre el municipium y el santuario.

\title{
2. TEXTO
}

\author{
DIANA[E AVG(ustae)] \\ COLLEG(ii) [LOTOR(um)] \\ S[ACR(um)] \\ PRIMIGE[NIVS R(ei) P(ublicae)] \\ ARICINO[RVM SER(vus) ARC(arius)] \\ CVRATOR[II CVM] \\ M ARRECIN[O GELLIANO] \\ FILIO CV[RATORE I] \\ $\mathrm{D} \quad[\mathrm{D}]$
}

\section{LA DEDICATORIA}

Como fácilmente puede percibirse, se trata de una dedicatoria a Diana por parte de dos curatores, padre e hijo, del collegium lotorum. Pero, las interpretaciones no son unánimes: Illuminati (1989: 35) y Diosono (2011) desarrollan COLLEG(ium) concertando con SACR(um) y Bruun (1993) COLLEG(ium) LOTOR(um) SACR(orum), lo cual convierte en "sagrados" bien sea al collegium bien sea a los lotores, una interpretación que condiciona desde el inicio ulteriores comentarios. Dado el significado que tiene en latín el adjetivo sacer,-a,-um como algo perteneciente a la esfera de lo divino, no parece posible calificar de sacrum un colegio profesional formado por esclavos y libertos. De hecho, el CIL y estudiosos como Granino Cecere 
(2000: 43, n. 27), Tran (2006: 53) o Vincenti (2010: 154-155) dan un desarrollo correcto. No obstante, hay que reconocer que la estructura sintáctica no es frecuente: "consagrado a la Diana Augusta del colegio de los lotores", una fórmula que crea un estrecho vínculo entre la diosa y el collegium, convirtiéndola así en su divinidad tutelar, una especie de "patrona" de los lotores. No es este un caso único de genitivos de este tipo y puedo aportar al menos un paralelo, datado en el año 128, (CIL VI 30901 = EDR029206 = Friggeri-Granino-Gregori, 2016: 482-483): Herculi domus Augusti sacrum, "consagrado al Hércules de la familia augusta". Es excepcional el epíteto Augusta aplicado a Diana, puesto que se trata del único caso en la regio I (Latium) (Gregori, 2009: 318); hay 4 ejemplos en Roma y 17 en toda Italia; las dedicatorias a las divinidades augusteas suelen realizarlas libertos, seviri angustales y gentes de clases inferiores. Aquí podemos preguntarnos el por qué del epíteto en Ariccia. ¿El collegium lotorum tuvo alguna relación con alguno de los sucesivos Augustos? ¿Fue creado o beneficiado por alguno de ellos o alguno tuvo relación con él? Sin entrar para nada en las relaciones de Calígula con el lago y el santuario ${ }^{3}$, conocemos las siguientes inscripciones imperiales procedentes de Nemi: una dedicatoria fragmentaria a Vespasiano (Granino Cecere 2000: 39-40, fig.8) (CIL XIV 4191 = EDR147217) por parte del [senatus pop] ulusque ari[cinorum], datada en el año 71 y conservada en Roma; la parte derecha de un arquitrabe (CIL XIV 2216 = EDR094036) de un edificio restaurado por Adriano en el año 121-122, conservada en el Museo delle Navi de Nemi; del mismo año (y quizás del mismo taller), una tábula de mármol completa dedicada al emperador también por parte del senatus populusque aricinus. (Eph. Epigr. IX 651 = EDR146750), conservada en la Ny Carlsberg Glyptotek de Copenhagen. A estas hay que añadir un pequeño y fragmentario epígrafe votivo (pro salute de Claudio y su familia) (Eph.Epi. VII 1242 = CIL XIV 4191 = EDR147609; Granino Cecere, 2000: 40), de carácter diferente, probablemente privado, que debe datarse en los inicios del año 50 y sobre el que volveremos más adelante. Tenemos pues testimoniados a Claudio, Vespasiano (quizás con sus hijos Tito y Domiciano) y también a Adriano, época a partir de la cual el culto a Diana en general adquirió un auge notable. Hay que mencionar también un fragmento perdido, (CIL XIV 2159 = EDR144083), según Lucidi (1796: 224-225) hallado en 1776 en Vallericia (no en las excavaciones de Monseñor Despuig, como se dice, sino en las de Gavin Hamilton), en el que se leía ....sari/...nnico/... claudeif.; Lucidi transcribe: ... audio/ ... saril ...nnicol ...claudiif.

\title{
4. LOS DEDICANTES
}

Son dos: Primigenius, $r(e i) p$ (ublicae) aricinorum servus arcarius (Silvestrini, 2005) es decir "administrador" o "contable" municipal, el encargado del arca, la "caja";

\author{
Ghini (ed.) 2013.
}


estos "funcionarios" manejaban notables cantidades de dinero y tenían una posición particular en la sociedad de su ciudad, hasta el punto de contraer matrimonio con personas de condición no servil, como quizás fuera el caso de nuestro hombre; Primigenius era además curator del collegium lotorum por segunda vez; en la dedicatoria asocia a su hijo, liberto, curator del collegium por primera vez; debemos observar la sutil diferencia entre ambos: el sujeto es el padre. Aunque el nombre de Primigenius no nos diga nada, al menos a mí, sus dos funciones evidencian una estrecha relación entre el municipium y el collegium, al menos en la época que nos ocupa, aunque quizás esto cambiara con el paso del tiempo. Esta duplicidad de funciones plantea la cuestión de la efectiva administración del santuario y sus bienes (Bodei Giglioni, 1977: 45-47), la organización del culto, etc., que debía depender de los magistrados y del personal del municipio. El nombre del hijo, M. Arrecinus Gellianus, es más sugerente. Se discute si el origen de la gens Arrecina (Demougin, 1978; Forci, 2011) hay que situarlo en Ariminum (Rímini) o en Pisaurum (Pésaro); no obstante, desde un punto de vista lingüístico Arrecinus deriva de Aricia (TLL, vol. II, s.u. Arrecinus) (arrecinus / aricinus) y además, Arrecinus es un liberto de la res publica aricinorum; por tanto habría que explicar de otro modo la presencia adriática de la gens y quizás recordar la antigua relación entre Ariminum y el santuario de Nemi (CIL I/2 $40=C I L$ XIV 4269) (Illuminati, 1989; Cicala, 1995). Aunque es un gentilicio muy poco frecuente, se encuentra también en Bovillae (Marino) (CIL XIV 2408 = EDR155784), a cuatro millas de Ariccia, y en Tusculum (CIL XIV 2679, libertos); durante el s. I hubo una gens Arrecina bien situada en las altas esferas del poder: M. Arrecinus Clemens (Eck, 1999) fue prefecto del pretorio en época de Calígula (año 38-41) y participó en la conjura para asesinarlo, pero se desconoce su destino posterior; Arrecina Tertulla, su hija, fue la primera y efímera esposa (año 62-63, en época de Nerón) del futuro emperador Tito (Suet. Tit.4), fallecida en el año 63, y su hijo, M. Arrecinus Clemens, fue también prefecto del pretorio (Tac. Hist. 4.68) en época de Vespasiano (año 70) y murió asesinado por Domiciano (Suet. Dom.11.2).

\section{EL COLLEGIVM LOTORVM}

Hay que partir del hecho de que es un unicum y de que desconocemos su naturaleza; los lotores no son cultores ni sodales, ni se trata de un collegium funeraticium, por tanto hay que pensar que sería un colegio profesional; sus dos curatores, los "encargados", no nos ilustran mucho. Lotor es un término inusual, sin paralelos en los textos literarios y apenas en los epigráficos; es un sustantivo agente (tipo auctor o cantor) del verbo lavo, is,-ere, lautum (TLL, vol. VII 2, s.u. lavo) y por tanto lotor (TLL, vol. VII 2, s.u. lavator) significa "el que lava" algo o a alguien. Ya Ligorio tradujo lotores por "lavatori”. ¿Los que lavan qué? ¿personas, ropa, telas, objetos cultuales? Pero, quizás, al menos en el caso de Ariccia-Nemi, cabría considerar otra posibilidad: que lotor fuera un agente del verbo luo, lui, luere (TLL, vol. VII 2, s.u. luo), - gr. $\lambda v ́ \omega$, "liberar" (Beekes,

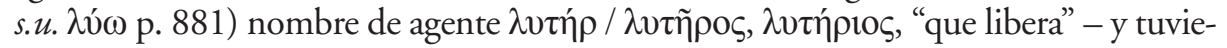
ra relación con el compuesto, muy conocido y usado en textos epigráficos, soluo, solui, soluere, solutum, "pagar", "expiar"; luo es un término del derecho (luere poenam) 
de uso poco frecuente, aunque se encuentra en Cicerón (Phil. 14.32, Illi igitur impii, quos cecidistis, etiam ad inferos poenas parricidii luent, ...), en los poetas augusteos (Verg. Georg. I.502, Laomedonteae luimus periuria Troiae, Aen. 1. 136, Post mibi non simili poena commisa luetis) y alguna vez en Livio, 10.28.13, Datum hoc nostro generi est ut luendis periculis publicis piacula simus. Iam ego mecum hostium legiones mactandas Telluri ac Dis Manibus dabo. (devotio de P. Decius en la batalla de Sentinum). Volveremos sobre el tema.

Como es lógico, me han precedido estudios varios sobre la cuestión y las distintas opiniones pueden resumirse así: ya Mancini (1911) consideró a los lotores como "addetti alle terme o bagni pubblici, che erano annessi al santuario di Diana nemorense" y los puso en relación con CIL XIV 4190 (= EDR147157), texto grabado en una columna y datable no antes del s. II (Granino Cecere, 2002: 34, fig.4), donde se hace referencia a un balneum vetus. Illuminati (1989) formuló la extraña hipótesis de que fueran los trabajadores de una fullonica que recogían los orines. Scheid (1991) conjugando la idea de "lavar" con la de "purificar", hablaba en términos generales de la purificación por el agua, de abluciones rituales, de la presencia de baños en santuarios extra-urbanos a los que los devotos llegaban tras un largo camino. En esta dirección va el trabajo de Bruun (1993), quien considera a los lotores como servidores de los baños, pero quizás de algún tipo especial, "perhaps for sacrificial purposes"; hay que tener en cuenta que el término lotor no aparece en el vocabulario termal (Nielsen, 1990; Rebuffat, 1991); existen innumerables termas, pero en ellas no hay lotores. Para Tran (2009:179) serían simples "blanchisseurs", "lavanderos", sin mayores explicaciones. Finalmente, Diosono (2011), en la línea de Bruun, opina que eran personal del santuario y al final de su trabajo los pone en relación con algunas "vasche", es decir "pilas o bañeras", halladas en las "celle donarie" (capillas para ofrendas) de la terraza inferior del santuario, lo cual es de sumo interés.

El término lotor no se encuentra en los textos literarios; de hecho el único pasaje que se aduce como ejemplo necesita un comentario: se le atribuye a Paulino de Nola (s. IV-v), Ep. 23.4, pero eso no es así; se trata de un texto de Agustín, C.D. 6.10, que reproduce un fragmento de Séneca (Estienne, 2001: 203, n. 70). Además, y esto me parece muy importante, en el pasaje hay un problema de crítica textual y el término lotor es una restitución. Por tanto, es más prudente no tomarlo como base de ninguna hipótesis. Otros textos que suelen citarse son muy tardíos, varios de ellos de latín cristiano, que dudo sirvan para solucionar un problema del s. I

En los textos epigráficos tan sólo se conocen cuatro testimonios más. Uno en la misma Ariccia, otro en Aquileia y otro en Altinum (junto a la laguna de Venecia, por tanto relativamente próximos) y el cuarto en Mauritania Caesariensis (Argelia). El segundo documento epigráfico que atestigua la existencia de un collegium lotorum en Ariccia-Nemi es un altar funerario (ILS 9421 = EDR072486), de mármol, datado hacia finales del s. I o inicios del s. II, hallado en 1885 (Mancini, 1911) en la "Olmata di mezzo" de Genzano (practicamente unido a Ariccia), a pocos metros de un tramo de lo que debía ser el clivus aricinus, es decir el ramal que desde la vía Apia conducía al santuario de Diana. Se conserva actualmente, muy estropeado (por haber sido grabado un crismón en el espejo epigráfico), en el cementerio de Genzano (Illuminati, 1989). El texto dice: Diis Manibus / L. Antonio Ionico / sodali ivvenum / colleg. Mart. Salut. / 
et quinq.colleg. lot. I nemorensium quing / Cornelia Thallusal coniug. suo ben. mer. f. I et sibi cum quo vix. a. XXX. Como vemos, se trata del epitafio de L. Antonius Ionicus, que fue sodalis iuvenum collegii Martis Salutaris y quinquennalis collegii lotorum nemorensium, al parecer cinco veces; era con toda probabilidad un liberto, lo mismo que su esposa. Hay aquí interesantes diferencias en relación al epígrafe de Diana Augusta: el collegium lleva el adjetivo de nemorense, en referencia al topónimo Nemus (Malaspina, 2000), lo cual prueba que sus miembros "ejercían" en el santuario, y ha desaparecido cualquier referencia a la res publica aricinorum. Esta es una de las escasísimas veces en que el adjetivo nemorensis-e aparece en la documentación epigráfica y, detalle muy importante, no se refiere a la divinidad sino a un collegium; es un adjetivo, no un epíteto de Diana.

El texto del ara de Aquileia $(C I L \vee 801=$ Brusin 1991-1993, no $301=$ EDR117429), conservada en el Museo Archeologico Nazionale y datada en el s. II, dice: Minervae / Aug. sacr. / M. Valerius / Venustus / et Mulcedatia Tais / gentilibus / artorianis lotoribus / aram d.d. Es muy difícil comprender qué función desempeñaban los gentiles artoriani lotores; en general se tiende a pensar que eran fullones o trabajadores de la lana (Zaccaria, 2009: 283), pero no hay una certeza ni un acuerdo. Más desconcertante es el cipo funerario de Altinum (NSA 1930, $479=A E$ 1931, $\mathrm{n}^{\circ} 98$ = EDR073173), datado en época augustea, en el que se lee $f($ ronte) $p$ (edes) XXX ab lotor $/ . . .7$ p(edes) XXX ab strat... (Buonopane, 2003: 287, fig.1.c).

El cuarto testimonio es una placa rota en dos fragmentos descubiertos con varios decenios de intervalo en Aïn Bessem (Thébert, 2003), cerca de Auzia (actual Sour El Ghozlane, en el interior de Argelia) (CIL VIII 9183 = 20281), que contiene un carmen (CLE 577) de diez versos; de cronología tardía, aunque sin precisión, el texto empieza : B alnea rura domus fec [it C]onstantinus auctor / E t fecit ut memore[nt c] ari de sanguine nati / $N$ am ut plene lotor [dis]cas quid sit perferre laborem / ... Las letras iniciales de los versos forman en acróstico Bene lavate o bene lava te.

A la vista de estos documentos uno se siente inclinado a pensar que es muy difícil encontrar un significado unitario para el término lotor que pudiera funcionar en todos los casos; también a pensar que lotor pudiera significar cosas distintas, aunque nos parezcan relacionadas. Esta idea me ha llevado de nuevo a la cuestión

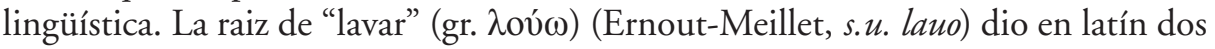
verbos, uno de la $1^{\text {a }}$ conj. Lauo, -as,-are, lauatum, con -a- larga, y otro de la $3^{\mathrm{a}}$ conj. Lauo, -is, lauere, laui, lautum, (lotum), con el cual se formaron gran número de compuestos en -luo, entre ellos abluo < ab-luno < ablauo (la vocal interior asume el timbre -ucuando le sigue una -u- consonántica). Por otra parte hemos visto que existe el verbo luo, -is, -ere, (gr. $\lambda v ́ \omega)$ "liberar", "expiar", poco utilizado y reemplazado por su compuesto soluo (< *se-luo), "pagar", "liberar", de ahí absoluo. Entre el sustantivo agente de lauo y el de luo la diferencia es mínima, lautor / lotor / lutor. Además, en los testimonios ariccino-nemorenses quizás convergen ambos significados, "liberar", "purificar" mediante el "lavado", la purificación por el agua; en realidad, se le añade un contenido "moral" a "lavar". Cabría la posibilidad de que se produjera una (con)fusión entre ambos verbos, lo cual explicaría expresiones como "lavar los pecados" en español o "laver une injure" en francés. 


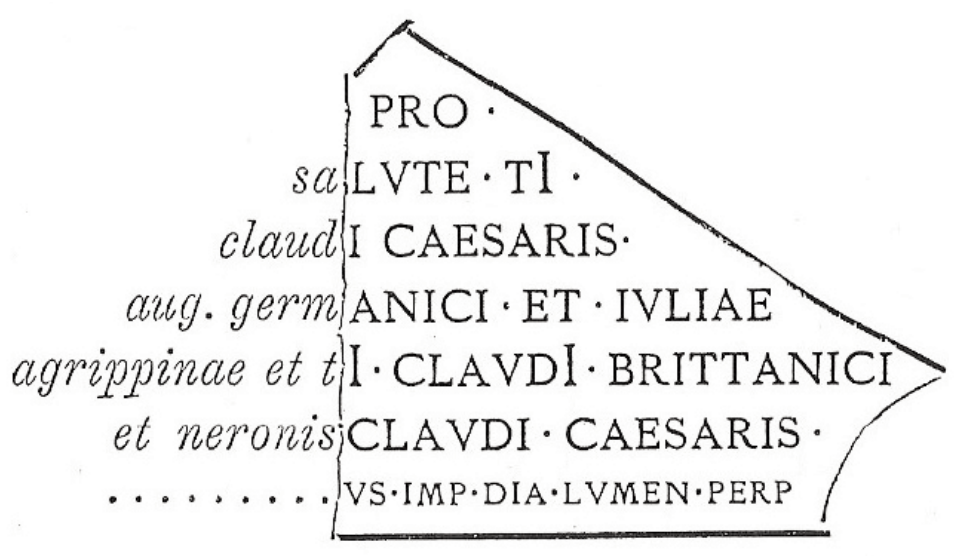

Fig. 2: CIL XIV 4191.

Dibujo tomado de NSc (Notizie degli Scavi di Antichità), 1888, p. 194.

Llegados a este punto, es lícito volver a plantearnos si pudo haber una relación entre el collegium lotorum y el epíteto aplicado a Diana, Augusta. Las inscripciones imperiales halladas en el área de Ariccia y Nemi no nos aportan gran información a excepción de la pequeña ofrenda por la salud de Claudio y su familia, así que vamos a dedicarle una cierta atención.

\section{LA INSCRIPCIÓN DE NEMI CIL XIV 4191 = EDR147609 (FIG. 2)}

El epígrafe, perdido, fue hallado en 1887 en las excavaciones realizadas por Luigi Boccanera; procede de una de las denominadas "celle donarie" (Braconi et alii, 2013: 24-28) adosadas a la parte noroccidental de la terraza inferior del santuario; sería muy interesante saber en cual de ellas, pero no he conseguido encontrar este dato con seguridad; Ghini dice (Braconi et alii, 2013: 24) que en la "cella $c$ vi erano alcune iscrizioni", sin precisar; es en este ambiente donde se construyó una pila con el interior revestido por revoque hidraúlico; también Diosono se refiere a estas "vasche". En este contexto sería conveniente recordar que en las excavaciones realizadas por Lord Savile, embajador inglés en Roma, (AA.VV., 1983: 11-14) en 1885 en la zona de las "celle donarie" se encontraron diversas estatuas y retratos (Ghini, 2013: 24): una estatua de Tiberio, de mármol (AA.VV., 1983: 21), otra de Druso y un retrato de Germánico; desconozco dónde se conservan, si es que existen; quizás en Estados Unidos, bien en The University of Pennsylvania Museum of Archaeology and Anthropology (PENN) o bien en el Museum of Fine Art de Boston; procedían de la colección del príncipe Orsini, propietario de Nemi en esa época y que se repartió los hallazgos con Lord Savile. Para el tema que nos interesa, no hay que pasar por alto el hecho de que se hallaran los retratos de Druso y Germanico, padre y hermano de Claudio. Volviendo a nuestra inscripción: se trataba de una pequeña tablilla (0’12 cm. alto x 0’22 ancho) de mármol blanco y forma de triángulo 
isósceles, de la cual sólo se conservaba algo más de la mitad en sentido vertical. Dada la forma y el tamaño, podría pensarse que estaría en el frontón de una pequeña capilla, o mejor una hornacina, donde habría colgada una lámpara protegida de la lluvia y demás inclemencias; por el dibujo publicado en NSc 1888, p.194 (fig. 2) a partir de un "calco cartaceo", el texto estaría distribuido en 7 líneas, la última con letras de menor tamaño y decía:

\author{
PRO \\ [sa]LVTE TI \\ [claud]I CAESARIS \\ [aug germ[ANICI ET IVLIAE/ \\ [agrippinae et t]I CLAVDI BRITTANICI (sic) \\ [et neronis] CLAVDI CAESARIS \\ [.....]VS IMP DIA LVMEN PERP
}

Observaciones: Agripina no lleva el título de Augusta; si el dibujo es fiel al original (y no hay razones para pensar que no lo sea) y hechos los cálculos, se constata que no hay espacio para AVG a no ser que se abreviara Agrippina, lo cual no sería correcto; este no es un caso único, ya que se conocen al menos otros dos, uno en Herculano $(C I L \times 1418$ = EDR107838) y otro en Pompeya $(C I L \times 933=$ EDR149259). Por otra parte, el nombre de Británico todavía precede al de Nerón, aunque parece que éste ya había sido adoptado por Claudio (25 febrero año 50). Según Tac. Ann. 12.26. rogataque lex qua in familiam Claudiam et nomen Neronis transiret. augetur et Agrippina cognomento Augustae, parecen haber sido dos hechos si no juntos, al menos muy próximos. Así que la tablilla habría que datarla entre la lex y la concesión del título de Augusta.

En la última línea figuraría en primer lugar el nombre del dedicante, del cual sólo nos queda la desinencia -VS; a continuación se lee IMP DIA, que en $N S c$, p. 194, (se repite en EDR147609) es desarrollado como IMP(erio) DIA(nae), "per monito della dea", aunque no creo que dicha propuesta sea correcta; "per monito" sería ex monitu (cf. CIL VI 134) o iussu (cf. CIL vI 30975, iussu Iovis) y Dia(nae) es un dativo, no un genitivo. Si intentamos imaginar la oración completa, tendríamos el sujeto al inicio de la línea y el complemento directo al final -lumen perp(etuum)-, el nombre de la diosa-Dia(nae) - y necesitaríamos un verbo, que sería IMP. No es fácil encontrar una propuesta, pero la comprensión de la frase podría venir dada por el objeto directo, "una lámpara permanente" que, se supone, estaría colgada en la hornacina, así que el verbo podría ser IMP(endet), de impendeo, "colgar". Recordemos a propósito el verso de Prop. 2.32.10, Triviae lumina ferre deae, citado al inicio. De todo ello resultaría la propuesta: pro salute Ti. Claudi Caesaris Augusti Germanici et Iuliae Agrippinae et Ti. Claudi Brittanici et Neronis Claudi Caesaris, [.....] imp(endet?) Dia(nae) lumen perp(etuum).

Es muy interesante la ofrenda de un lumen perpetuum, una luz perpetua, probablemente con mechas de fibra de amianto (Plin. N.H. 36.139, amiantus alumini similis nibil igni deperdit); al inicio me he referido a la procesión de antorchas en los idus de agosto; citar también en este contexto la cantidad de lucernas halladas en las aguas del lago (Diosono-Cinaglia, 2016). 
El epígrafe de Claudio y su familia nos lleva de modo inevitable a considerar un pasaje de Tácito; la asociación de ambos documentos, literario y epigráfico, no es mía, sino que se debe a Momigliano (1966: 648), aunque él no la llevó más lejos. También Granino Cecere (2000: 40) establece la relación. El día de la boda de Claudio con Agripina, hija de su hermano Germánico, L. Junio Silano se suicidó, acusado de incesto con su hermana Junia Calvina (Pasqualini, 2001 [2013], quien no relaciona texto y epígrafe). ¿Quién era Silano? Era el prometido de Octavia (Agripina habría deseado su boda con Nerón, como así sucedió en el 53), la hija de Claudio y Mesalina, nieto por vía materna de Julia Menor y por tanto descendiente de Augusto; su padre, Apio Junio Silano, que había sido gobernador de la Tarraconense y estaba casado con Emilia Lépida, la madre de Mesalina, ya había sido condenado a muerte por Claudio en el año 42-43 (Suet. Cl. 29); un lío de familia muy al estilo de los JulioClaudios. He aquí el relato de Tácito, Ann. 12.8.1: Die nuptiarum, Silanus mortem sibi conscivit, sive eo usque spem vitae produxerat, seu delecto die angendam ad invidiam. Calvina, soror eius, Italia pulsa est. Addidit Claudius sacra ex legibus Tulli regis piaculaque apud lucum Dianae per pontifices danda, inridentibus cunctis, quod poenae procurationesque incesti id temporis exquirerentur. "El día de la boda Silano se suicidó, ya sea porque había mantenido hasta entonces la esperanza de vida, ya sea para aumentar lo odioso del hecho eligiendo ese día. Su hermana Calvina fue expulsada de Italia. Claudio añadió ceremonias sagradas y ceremonias expiatorias según las leyes del rey Tulio que habían de ser celebradas por medio de los pontífices en el bosque sagrado de Diana; todos se reían de que se pidieran penas y expiaciones por un incesto en aquel momento". Observemos que pontifices no es el agente de danda, sino que los piacula debían ser ofrecidos per pontifices, "por medio de los pontífices", los miembros del colegio sacerdotal más importante de Roma. ¿Algunos pontífices se desplazaron a Nemi? Mucha pompa por alguien que había sido expulsado del ordo senatorius (Tac. Ann. 12.4) y obligado a suicidarse (Suet. Cl.29. Silanus abdicare se praetura ante IIII Kal. Ian. morique initio anni coactus die ipso Claudio et Agrippinae nuptiarum.); Suetonio incluso dice que fue obligado a morir ese mismo día, lo cual lo convertiría en una especie de "víctima propiciatoria". El mismo Tac. Ann. 12.5, nos explica los temores de Claudio sobre su matrimonio con la hija de su hermano: C. Pompeio Q. Veranio consulibus (año 49), pactum inter Claudium et Agrippinam matrimonium iam fama, iam amore inlicito firmabatur, necdum celebrare sollemnia nuptiarum audebant, nullo exemplo deductae in domum patrui fratris filiae: quin et incestum ac, si sperneretur, ne in malum publicum erumperet metuebatur. Claudio temía que, si cometía un incesto, acabara en un mal público. De hecho, hizo votar al senado un decreto para "despenalizarlo": Suet. Cl. 26. subornavit proximo senatu qui

${ }^{4}$ En la Apocolocintosis, 11.5. figuran padre e hijo entre las víctimas de Claudio: Quando quidem divus Claudius occidit socerum suum Appium Silanum, generos duos Magnum Pompeium et L. Silanum...; 10.4: ... ut duas Iulias proneptes meas occideret, ... unum abnepotem L. Silanum. 
censerent, cogendum se ad ducendum eam uxorem, quasi rei p. maxime interesset, dandamque ceteris veniam talium coningiorum, quae ad id tempus incesta habebantur. Ac vix uno interposito die confecit nuptias. Tac. Ann. 12.7. senatumque ingressus decretum postulat quo iustae inter patruos fratrumque filias nuptiae etiam in posterum statuerentur. En cierto modo parece una contradicción con la acusación a Silano, pero no es así; sólo se "despenalizaban" las bodas entre tíos y sobrinas, no las relaciones entre hermanos. Si he entrado en los pormenores del caso es porque me parece que ayudan a comprender el trasfondo del asunto.

Como es lógico, el texto de Tácito, 12.8, ha suscitado variados comentarios: lucus Dianae ¿el Aventino o Nemi? Ex legibus Tulli regis ¿Tullus Hostilius o Servius Tullius? Es cierto que Tácito no habla expresamente del santuario de Nemi, pero la expresión lucus Dianae hace pensar en ello, ya que no hay referencias a un lucus en el Aventino y menos en esta época; aunque bien es cierto que no se ha llegado todavía a una definitiva, convincente y segura ubicación del templo de Diana en el Aventino (Venditelli, 1995; Scodellari, 2003; Armellin-Quaranta, 2004; Marcattili, 2012) ni en su fase arcaica (s. VI a.C.) ni en la reconstrucción de L. Cornificius en época augustea (Res Gestae, 19.2; Suet. Aug. 29.5). El otro motivo de discusión es la referencia ex legibus Tulli regis, el rex Tullus / Tullius ha sido identificado preferentemente con Tulo Hostilio (Pasqualini, 2001; Moreau, 2002: 47-49) y en pocos casos con Servio Tulio (Momigliano, 1966). En realidad rey y santuario van estrechamente unidos: Servio Tulio nos lleva al Aventino ya que él fue el fundador del templo allí dedicado a Diana (Livio, 1.45; D.H. 4.26.5); Tulo Hostilio no nos lleva directamente al bosque de Nemi, pero si nos lleva a los Colli Albani, ya que el hecho más relevante de su reinado fue la guerra y posterior destrucción de Alba Longa, cuya localización se supone no lejos de Ariccia y del lago.

Voy a proponer aquí una hipótesis basada en el contexto histórico del pasaje de Tácito, la boda de Claudio con Agripina, tras la muerte de Messalina, cuando Claudio tenía ya casi 60 años. Silano se suicidó el mismo día para augendam ad invidiam, para aumentar lo odioso del hecho, para hacer coincidir el "castigo" por un (supuesto) incesto con la consumación solemne de otro. Tácito se refiere a leges del rey Tulio; es cierto que D.H. 4.26. habla de leyes (vó $\mu o r)$ de Servio Tulio en el templo de Diana, pero eran leyes sobre las asambleas federales, etc.; el mismo Tácito, Ann. 3.26. dice praecipuus Servius Tullius sanctor legum fuit, pero no hay referencias a leyes de carácter religioso en su actividad legislativa. En cambio, en el relato de Livio, 1.26. sobre el reinado de Tulo Hostilio hay un pasaje -y una frase- que puede resultar revelador: estamos al final del muy conocido episodio de los Horacios y los Curiacios (cuyo supuesto y grandioso sepulcro (Coarelli, 1981: 92-93), construido en la primera mitad del s. I a.C., se encuentra a una milla de Ariccia; aunque hay otro complejo sepulcral atribuido a los hermanos en la quinta milla de la via Apia (Coarelli, 1981: 52-55); el Horacio superviviente regresa vencedor a Roma pero se encuentra con su hermana que llora a su prometido, uno de los Curiacios muertos; entonces comete un atrox facinus, la mata. El rey Tulo Hostilio lo acusa de perduellio, "alta traición", porque el crimen recae sobre toda la comunidad y dicta una lex terrible. En cierto modo la historia acaba bien (si así puede considerarse): Horacio es absuelto 
por el pueblo, sensible a las lágrimas del padre y al valor del hijo. Pero... ut caedes manifesta aliquo tamen piaculo lueretur, imperatum patri ut filium expiaret pecunia publica. Is, quibusdam piacularibus sacrificiis factis, ...; era preciso expiar el crimen con algún sacrificio de purificación. Livio no nos explica qué tipo de piaculum debía realizarse ni dónde debía llevarse a cabo; si el lugar fuera el lucus Dianae, estaríamos remontando al s. VII a.C. el culto en un lucus a orillas del lago, lo cual nos llevaría a los materiales cerámicos protohistóricos hallados en la terraza superior del santuario, próximos a un manantial y al llamado "ninfeo de Egeria" (Ghini et alii, 2015; Diosono, et alii, 2019). Interesante también que el piaculum debiera hacerse pecunia publica. En el texto de Livio encontramos el verbo luo, del que antes hemos hablado, el término preciso para expiar un delito. Claudio, el princeps "anticuario" (Huzar, 1984) que había pasado más de media vida dedicado a escribir libros (en griego) sobre los etruscos, sobre Cartago y sobre antigüedades varias (Suet. Cl. 42.20), debía conocer mucho mejor que nosotros este tipo de tradiciones y de rituales. Cierto que no es lo mismo matar a una hermana que casarse con una sobrina pero para los romanos el scelus, el acto impuro, no estaba en la naturaleza de la acción sino en la consanguinidad y constituía un mal público, que afectaba a la colectividad. En realidad, que había una relación entre Ariccia y la leyenda de los Horacios lo prueba el hecho de que la ciudad estaba inscrita en la tribu Horatia (Taylor, 1960: 43; Granino Cecere - Ricci, 2010: 151-152), lo cual me parece notablemente importante.

Ahora volvemos a Silano y a los piacula por el supuesto incesto con su hermana; en realidad, se trataba de una expiación "por procuración"; poco después de celebrar su boda en Roma, Claudio expiaba en cierto modo su incesto en Nemi; quizás nos pueda parecer una farsa, pero quizás no lo fuera; así se comprendería mejor que los piacula se celebraran lejos de la Urbs. ¿¿ra este el único motivo o pudo haber otros? Cierto que Diana pasaba por ser una diosa "casta", $\pi \alpha \rho \theta \dot{v}$ os (Himno Homérico a Artemis, v. 2; Calímaco, Himno a Artemis, v. 110), invocada en ocasiones como Virgo (CIL II 2660; CIL IX 5796), pero desconozco que tuviera poderes "purificadores".

¿Qué tipo de piacula fueron ofrecidos a Diana para purificar el incesto? Todo lo que digamos son puras especulaciones, pero dado el lugar, sería lógico que fueran ceremonias con intervención del agua, no sólo del lago, no de unos balnea que en época arcaica no existirían, sino de agua "viva", agua de alguno de los diversos manantiales que entonces como ahora, brotan en el bosque y en el entorno; no olvidemos la leyenda de la ninfa Egeria, esposa y consejera de Numa Pompilio, el antecesor de Tulo Hostilio, trasladada al lucus Dianae después de la muerte del rey (Ov. Met. 15.485 ss.; Fast. 3.261ss.; Pena - Oller, 2012) y convertida en el numen de un manantial.

Llegados a este punto debemos preguntarnos si es posible -y correcto desde un punto de vista metodológico- establecer una relación, a través del epíteto Augusta y quizás también del dedicante, entre ambos epígrafes, entre el collegium lotorum y las ceremonias para purificar un incesto celebradas en el año 50; es evidente que aquí entramos en el terreno de las hipótesis, puesto que, hoy por hoy, es imposible demostrar que el collegium fuera creado precisamente en esta ocasión para agrupar y organizar a los "asistentes", "ayudantes", o algo similar, de los pontifices en los piacula y que luego continuarían ejerciendo en circunstancias menos excepcionales; los lotores 
no serían simplemente "los que lavan" sino "los que purifican"; se trata de una insólita denominación (que yo sepa, no se conoce ningún otro collegium lotorum en ningún otro santuario) para una función poco habitual. La cronología de ambos epígrafes no impide relacionarlos; aunque ignoro durante cuánto tiempo solía ejercer un curator ni cuánto tiempo pudo transcurrir entre la primera y la segunda curatio de Primigenius, la dedicatoria a Diana Augusta podría ser a lo sumo un decenio posterior, es decir hacia el año 60. Importante sería conocer la datación de las "vasche" construidas en algunas de las "celle donarie".

Aunque aceptáramos la hipótesis propuesta, diversos interrogantes subsistirían: ¿por qué Primigenius, el servus arcarius? ¿Los piacula debían ser sufragados pecunia publica y para esto podía servir el arca del municipium aricinum? ¿Quién y por qué dedicó la plaquita pro salute Claudi y el lumen perpetuum? ¿Un devoto cualquiera? Hay un detalle curioso y posiblemente casual: en el dibujo de $N S_{c}$ (fig. 2), en el espacio de la última línea donde debía estar el nombre del dedicante encaja sin problemas el nombre de PRIMIGENI]VS, 9+2, once espacios. Otra cuestión que, en mi opinión, queda en la sombra, es el nombre del hijo de Primigenius ¿un liberto del municipio o un liberto de los Arrecint? No se sabe nada de M. Arrecinus Clemens después del asesinato de Calígula, pero es posible que no quedara muy alejado de los círculos del poder si en el año 62 casó con el hijo mayor de Vespasiano a su hija, nacida durante el reinado de Claudio. Son circunstancias que hoy por hoy no podemos saber.

Para concluir, unas breves consideraciones sobre la purificación por el agua, que de un modo u otro existe en las distintas religiones: el baño ritual hindú en el Ganges, en Allahabad en el norte de India; las abluciones islámicas antes de la oración; y también el Cristianismo, en el que el bautismo (de $\beta \alpha \pi \tau i ́ \zeta \omega$, "sumergir") de Jesús por inmersión en el río Jordán marca el inicio de su vida pública. Desconozco la existencia de ceremonias de purificación por el agua en la religión romana; no obstante, se pueden rastrear una serie de testimonios interesantes: Verg. Aen. 2.717720, Tu, genitor, cape sacra manu patriosque Penates; / me bello e tanto digressum et caede recenti / attrectare nefas, donec me flumine vivo / abluero; tras una noche de destrucción y muerte, Eneas debe purificarse antes de emprender el viaje hacia su destino en Occidente. También la conocida leyenda (Liv. 1.45) del sabino que pretendía sacrificar una hermosa vaca a Diana en el Aventino y a quien el antistes le dirigió estas palabras: Quidnam tu, hospes, paras? Inquit, inceste sacrificium Dianae facere? Quin tu ante vivo perfunderis flumine? Infima valle praefluit Tiberis. Otro ejemplo: Ov. Fast. 5, 673682, Est aqua Mercurii portae vicina Capenae; / si iuvat expertis credere, numen habet. I huc venit incinctus tunica mercator et urna / purus suffita, quam ferat, haurit aquam. / uda fit hinc laurus, lauro sparguntur ab uda / omnia quae dominos sunt habitura novos. / spargit et ipse suos lauro rorante capillos, et peragit solita fallere voce preces: / ablue praeteriti periuria temporis, inquit, / ablue praeteritae perfida verba die... Se trataría de un manantial con un supuesto poder divino; con una rama de laurel empapada en ella, el mercader se salpica a sí mismo y a sus mercancías y ruega a Mercurio "limpia los perjurios del tiempo pasado, limpia las pérfidas palabras..." Sabemos que la purificación por el agua puede ser por inmersión, por ablución, por aspersión... No tenemos ni idea en qué consistiría el ritual de Nemi. 
Si la hipótesis aquí presentada tuviera visos de ser acertada, si los lotores fueran unos servidores del santuario dedicados a baños (o inmersiones) rituales purificadores, esto enriquecería y ampliaría nuestro conocimiento no sólo sobre las esperanzas y los ruegos para el porvenir con que los devotos peregrinaban al "espejo de Diana", sino también sobre las "faltas" del pasado de las que esperaban ser "absueltos", aunque no fueran necesariamente un incesto o una muerte. Su purificación impediría que su falta recayera sobre su familia o sobre su comunidad.

\section{BIBLIOGRAFÍA}

La bibliografía sobre algunos de los temas aquí mencionados es enorme, desbordante, a veces contradictoria y en ocasiones repetitiva, tanto sobre el santuario de Nemi como sobre Servio Tulio y el templo del Aventino. Aquí no aparece más que la utilizada; el lector encontrará en ella las referencias necesarias y la historia de todas las polémicas en torno a problemas hasta ahora insolubles.

AA.VV. (1983): Mysteries of Diana. The antiquities from Nemi in Nottingham Museums, Nottingham.

Armellin, P. - Quaranta, P. (2004): "Il tempio di Diana sull'Aventino. Nuove acquisizioni”, Bullettino della Commissione Archeologica Comunale di Roma CV (105): 279-298.

BEEKES, R. (2010): Etymological Dictionary of Greek, Leiden, Brill.

Bodei Giglioni, G. (1977): “Pecunia fanatica. Lincidenza economica dei templi laziali”, RSI 89/1: 33-76.

Braconi, P. - Coarelli, F. - Diosono, F. - Ghini, G. (2013): Il santuario di Diana a Nemi. Le terrazze e il ninfeo. Scavi 1989-2009, Roma.

Brusin, G. (1991-1993): Inscriptiones Aquileiae, 3 vols., Udine.

BRUUn, C. (1993): “Lotores: Roman Bath-Attendants”, ZPE 98: 222-228.

BuOnOpane, A. (2003): "La produzione tessile ad Altino: le fonti epigrafiche" en G. DI CRESCI MARRONE M. TIRELl (eds.), Produzione, merci e commerci in Altino preromana e romana, Roma, pp. 285-297.

Chantraine, P. (1968): Dictionnaire étymologique de la langue grecque, París.

Cicala, V. (1995): “Diana ariminense: tracce di religiosità politica” en A. CALBI - G. SUSINI (eds.), Pro poplo ariminese, Faenza, pp. 355-365.

Coarelli, F. (1981): Dintorni di Roma. Guide archeologiche Laterza, Bari.

Cogitore, I. (1992): "Séries de dédicaces italiennes à la dinastie julio-claudienne", MEFRA 104/2: $817-870$.

Crawford, M. (1974): Roman Republican Coinage, Cambridge (= RRC).

Demougin, S. (1978): “Un nouveau membre de la gens Arrecina”, MEFRA 90/1: 317-330.

Diosono, F. (2011): "Il collegio dei lotores ed i balnea del santuario di Diana Nemorensis", Bollettino dell'Unione Storia ed Arte 103:. 78-82.

Diosono, F. - Cinaglia, T. (2016): "Light on the water: ritual deposit of lamps in Lake Nemi”, JRA 29: 451-468.

Diosono, F. et alii (2019): "Le prime fasi edilizie del tempio di Diana a Nemi” en F. M. CIFARELLI S. Gatti - D. Palombi, Oltre "Roma Medio-Repubblicana”, Roma, pp. 383-390. 
ECK, W. (1999): “Arrecinus Clemens” en Der Neue Pauly, vol. 2, col. 26, Stuttgart.

Ernout, E. - Meillet, A. (1939, 2a ed.): Dictionnaire étymologique de la langue latine, París, 1939.

Estienne, S. (2001): "Les “dévots” du Capitole. Le "culte des images” dans la Rome impériale, entre rites et superstition", MEFRA 113/1: 189-210.

FrAZER, J.G. (1944): La rama dorada, México [traducción de la edición inglesa abreviada].

ForCI, A. (2011): "L'epigrafe di Bau Tellas (Senorbì-Cagliari): prime attestazioni della gens Arrecina e del culto di Liber Pater in Sardegna" en A. ForCI, L'epigrafe di Marcus Arrecinus Helius, Senorbì (Cagliari), pp. 29-59.

Friggeri, R. - Granino Cecere, M. G. - Gregori, G. L. (2012): Terme di Diocleziano: la collezione epigrafica, Milán.

Garofalo, P. (2014): "Non solo funerali: considerazioni sulle funzioni del collegio salutare di Lanuvio (CIL XIV 2112) e sulle ragioni della sua dedica a Diana e Antinoo" en Sulle rive dell'Acheronte. Costruzione epercezione della sfera del post-mortem nel Mediterraneo antico, vol. 2, Roma, pp. 65-74.

GHINI, G. (ed.) (2013): Caligola. La trasgressione al potere, Catalogo della mostra, Roma.

Ghini, G.- Diosono, F.- Braconi, P. (2015): "La dea del lago", Archeo 368: 34-53.

Granino Cecere, M. G. (2000): "Contributo dell'epigrafia per la storia del santuario nemorense" en J. R. Brandt - L. Tovati - J. Zahle (eds.), Nemi - Status quo. Recent Research at Nemi and the Sanctuary of Diana, Roma, pp. 35-44.

Granino Cecere, M. G. (2005): Latium Vetus 1., CIL XIV; Eph. Epigr. VII e IX. Latium Vetus praeter Ostiam, Roma. Supplementa Italica. Imagines.

Granino Cecere, M. G. - RicCI, C. (2010): "Le tribù del Latium vetus", XVI Rencontre sur l'épigraphie, Bari, pp. 151-155.

Granino Cecere, M.G. - Nonnis, D. - Ricci, C. (2017): "Epigrafia e culti nell'ager aricinus", en S. Antolini - S. M. MARENGO - G. PACI (EDS.), Colonie e municipi nell'era digitale. Documentazione epigrafica per la conoscenza delle città antiche (Macerata 2015), Macerata, pp. 267-300.

GREGORI, G.-L. (2009): "Il culto delle divinità auguste in Italia: un'indagine preliminare" en J. BODEL M. KajaVA (eds.), Dediche sacre nel mondo greco-romano, Roma, pp. 307-330.

GuASCO, F.E. (1775): Musei Capitolini Antiquae Inscriptiones, 3 vols., Roma.

HuZAR, E. (1984): "Claudius. The erudite emperor", $A N R W$ II 32,1: 611-650.

IlluminaTI, A. (1989): "Lotores Nemorenses", Documenta Albana (II serie) 11: 31-43.

LEFEVRE, R. (1977): Le antichità di Ariccia. Scavi e ritrovamenti archeologici dal XVIII al XX secolo, Roma.

LIGORIO, P. (2008): Libri delle iscrizioni latine e greche, vol. 7: libri XXXIV-XXXVIII. Codice XIII B7, Roma, [S. ORLANDI, ed.].

Lilli, M. (2002): Ariccia. Carta archeologica, Roma.

LUCIDI, E. (1796): Memorie storiche dell'antichissimo municipio ora terra dell'Ariccia e delle sue colonie Genzano e Nemi, Roma.

Malaspina, E. (1994-95): "Diana Nemorensis vs. Diana Aventinensis: priorità cronologica e paradigmi storiografici", Documenta Albana (II serie) 16-17: 15-35.

Malaspina, E. (2000): "Nemus come toponimo dei Colli Albani e le differentiae verborum tardoantiche" en J. R. Brandt - L. Tovati - J. Zahle (eds.), Nemi-Status quo. Recent Research at Nemi and the Sanctuary of Diana, Roma, pp. 145-152. 
ManCINI, G. (1911): “Genzano di Roma - Tratto di antica via e cippo sepolcrale scoperto nell'Olmata di mezzo", NSc 6: 265-266.

Marcattili, F. (2012): “Per un'archeologia dell'Aventino: i culti della media Repubblica”, MEFRA 124/1: 109-122.

Momigliano, A. (1966): "Sacra ex legibus Tulli regis", appendice a "Sul dies natalis del santuario federale di Diana sull'Aventino”, en A. MomigliAno, Terzo contributo alla storia degli Studi Classici e del Mondo Antico, Roma, pp. 647-648.

Moreau, Ph. (2002): Incestus et prohibitae nuptiae, Les Belles Lettres, París.

NieLSEN, I. (1990): Thermae et balnea: the architecture and cultural history of Roman public baths, 2 vols., Aarhus.

ORLANDI, S. (ed.) (2008): P. Ligorio, Libri delle iscrizioni latine e greche, vol. 7: libri XXXIV-XXXVIII. Codice XIII, Roma, De Luca.

PASQUALINI, P. (2006): "La latinità di Ariccia e la grecità di Nemi. Istituzioni civili e religiose a confronto", Annali I: 29-38.

Pasqualini, A. (2001 [2013]): "L'incesto di Silano e il bosco di Diana (Tac. Ann. 12.8.2)", Analecta Romana Istituti Danici 27: 141-149 = Latium Vetus et Adiectum: ricerche di storia, religione e antiquaria, Tivoli, pp. 191-2 [Themata 13].

PASQUAlinI, A. (2017): “Nuovi spunti su Diana (e Artemide) a Roma e a Nemi”, Civiltà romana Iv: 1-16.

PENA, M. J. (1973): “Artemis-Diana y algunas cuestiones en relación con su iconografía y su culto en Occidente", Ampurias, XXXV: 109-134.

PENA, M. J. (2018): “CIL XIV 2213 y las magistraturas del municipium aricinum (Lacio)”, Anuari de Filologia. Antiqua et Mediaevalia 8 (Miscelánea Mayer): 719-734.

Pena, M. J. - Oller, M. (2012): "Hipólito y Orestes en el santuario de Diana en Nemi: contaminaciones mitográficas antiguas y modernas. Análisis crítico de las fuentes literarias", Latomus 71/2: 338-372.

Petrucci, F. (2001): "Liscrizione dei Lotori di Diana nel Palazzo Chigi di Ariccia”, Castelli Romani, anno XLI, gennaio-febbraio, pp. 3-7.

ReBuffat, R. (1991): "Vocabulaire thermal" en Les thermes romains, Ecole Française de Rome, Roma, pp. 1-34.

SCODELlari, F. (2003): "Le temple servien de l'Aventin: essai de réinterprétation des données traditionnelles", Athenaeum 91: 417-433.

SCHEID, J. (1991): "Sanctuaires et thermes sous l'Empire" en Les thermes romains, Ecole Française de Rome, Roma, pp. 205-214.

SilvestrinI, M. (2005): "Gli arcarii delle città", MEFRA 117/2: 541-554.

TAYLOR, L. R. (1960): The voting districts of the Roman Republic, Roma.

THÉBERT, Y. (2003): Thermes romains d'Afrique du nord et leur contexte méditerranéen: études d'histoire et d'archéologie, Roma [BEFAR 315].

Thesaurus Linguae Latinae (TLL), Munich.

Tran, N. (2006): Les membres des associations romaines: le rang social des "collegiati" en Italie et en Gaules sous le Haut-Empire, Roma [Col. EFR 367].

Vendittelli, L. (1995): “Diana Aventina, aedes”, en Lexicon Topographicum Vrbis Romae (LTVR) II, Roma, pp. 11-13. 
Vincenti, M.C. (2010): Diana. Storia, mito e culto della grande dea di Aricia, Roma.

ZACCARIA, C. (2009): "Novità sulla produzione lanaria ad Aquileia. A proposito di una nuova testimonianza di purgatores" en M. G. Angeli Bertinelli - A. DonATI (eds.), Opinione pubblica e forme di comunicazione a Roma: il linguaggio dell'epigrafia, Faenza, pp. 277-298. 